\title{
Design of Quantum Dot-Nanowire Single-Photon Sources that are Immune to Thermomechanical Decoherence
}

Artioli, Alberto; Kotal, Saptarshi; Gregersen, Niels; Verlot, Pierre; Gérard, Jean-Michel; Claudon, Julien

Published in:

Physical Review Letters

Link to article, DOI:

10.1103/physrevlett.123.247403

Publication date:

2019

Document Version

Publisher's PDF, also known as Version of record

Link back to DTU Orbit

Citation (APA):

Artioli, A., Kotal, S., Gregersen, N., Verlot, P., Gérard, J-M., \& Claudon, J. (2019). Design of Quantum DotNanowire Single-Photon Sources that are Immune to Thermomechanical Decoherence. Physical Review Letters, 123(24), [247403]. https://doi.org/10.1103/physrevlett.123.247403

\section{General rights}

Copyright and moral rights for the publications made accessible in the public portal are retained by the authors and/or other copyright owners and it is a condition of accessing publications that users recognise and abide by the legal requirements associated with these rights.

- Users may download and print one copy of any publication from the public portal for the purpose of private study or research.

- You may not further distribute the material or use it for any profit-making activity or commercial gain

- You may freely distribute the URL identifying the publication in the public portal 


\title{
Design of Quantum Dot-Nanowire Single-Photon Sources that are Immune to Thermomechanical Decoherence
}

\author{
Alberto Artioli, ${ }^{1, \dagger}$ Saptarshi Kotal, ${ }^{1, \dagger}$ Niels Gregersen $\odot,{ }^{2}$ Pierre Verlot, ${ }^{3}$ Jean-Michel Gérard $\odot,{ }^{1}$ and Julien Claudon $\circledast^{1, *}$ \\ ${ }^{1}$ Univ. Grenoble Alpes, CEA, IRIG, PHELIQS, "Nanophysique et semiconducteurs" group, F-38000 Grenoble, France \\ ${ }^{2}$ DTU Fotonik, Department of Photonics Engineering, Technical University of Denmark, DK-2800 Kongens Lyngby, Denmark \\ ${ }^{3}$ School of Physics and Astronomy, University of Nottingham, Nottingham NG7 2RD, United Kingdom
}

(Received 8 March 2019; published 13 December 2019)

\begin{abstract}
Nanowire antennas embedding a single quantum dot (QD) have recently emerged as versatile platforms to realize bright sources of quantum light. In this theoretical work, we show that the thermally driven, lowfrequency vibrations of the nanowire have a major impact on the QD light emission spectrum. Even at liquid helium temperatures, these prevent the emission of indistinguishable photons. To overcome this intrinsic limitation, we propose three designs that restore photon indistinguishability thanks to a specific engineering of the mechanical properties of the nanowire. We anticipate that such a mechanical optimization will also play a key role in the development of other high-performance light-matter interfaces based on nanostructures.
\end{abstract}

DOI: 10.1103/PhysRevLett.123.247403

Bright sources of indistinguishable photons are key resources for photonic quantum information technologies [1]. In this context, semiconductor self-assembled quantum dots (QDs) offer important assets to realize practical devices. However, their solid-state environment also raises significant challenges, since the coupling of the QD to uncontrolled degrees of freedom may degrade the spectral purity of the emitted photons [2]. Despite impressive progress [3-8], it remains crucial to investigate QD decoherence channels. Among these, the unavoidable coupling to lattice vibrations has been intensively studied, and the impact of bulk acoustic phonons is now well understood [9-17]. Yet, modern quantum light sources are often based on a photonic nanostructure that defines a controlled electromagnetic environment around the QD [18]. Such a structuration necessarily reshapes the phonon landscape and gives rise to a discrete set of low-frequency mechanical resonances, which confine elastic energy in tiny volumes. Even at cryogenic temperature, the thermal excitation of a single mechanical mode can then have a sizable influence on the QD optical linewidth. This was demonstrated very recently on a QD embedded in a nanowire antenna [19], a system widely employed to realize bright sources of quantum light [20-26]. These initial experimental results unveil a previously overlooked QD decoherence channel. While a few theoretical works have investigated the decoherence of a QD coupled to phonon continua of reduced dimension [27,28], the impact of the discrete phonon resonances hosted by realistic nanowire structures remains to be explored.

In this Letter, we show that low-frequency thermal vibrations constitute a major source of decoherence in QD-nanowire antennas, which prevents the emission of indistinguishable photons at liquid helium temperature.
A comprehensive theoretical analysis reveals that the QD spectral broadening is dominated by the contribution of a finite set of low-frequency mechanical modes, and strongly depends on the QD location within the nanowire section. To overcome this fundamental limitation, we propose several designs based on the engineering of the nanomechanical properties. We anticipate that such a mechanical optimization will play a key role in the development of other high-performance light-matter interfaces based on nanostructures.

Nanowire antennas exploit a tapered dielectric waveguide to shape the emission of a QD into a directive freespace beam [29]. As shown in Fig. 1, the nanowire features a diameter $d \sim \lambda / n$ around the QD, where $\lambda$ is the freespace operation wavelength and $n$ the nanowire refractive index. To optimize light collection efficiency, the nanowire stands on a bottom dielectric-metallic mirror and is terminated by a top taper, which can feature either a needle [panel (a)] or trumpet shape [panel (b)]. Needle antennas can be obtained with top-down [20,23] or bottom-up $[21,24]$ fabrication strategies, while photonic trumpets have so far been produced only through top-down processing [22]. In this work, we consider GaAs antennas $(d=200 \mathrm{~nm})$ which embed a self-assembled InAs QD $(\lambda=900 \mathrm{~nm})$. The emitter is located on the first antinode of the vertical standing wave pattern generated by the mirror reflection, $100 \mathrm{~nm}$ above the bottom mechanical clamping plane. This choice maximizes the optical bandwidth of the antenna, which exceeds $100 \mathrm{~nm}$ [30].

After an excitation in $|e\rangle$, the QD spontaneously relaxes to the ground state $|g\rangle$ by emitting a photon. To determine the photon spectral content, we build on the formalism developed in Ref. [11] and recently used in Ref. [28]. 

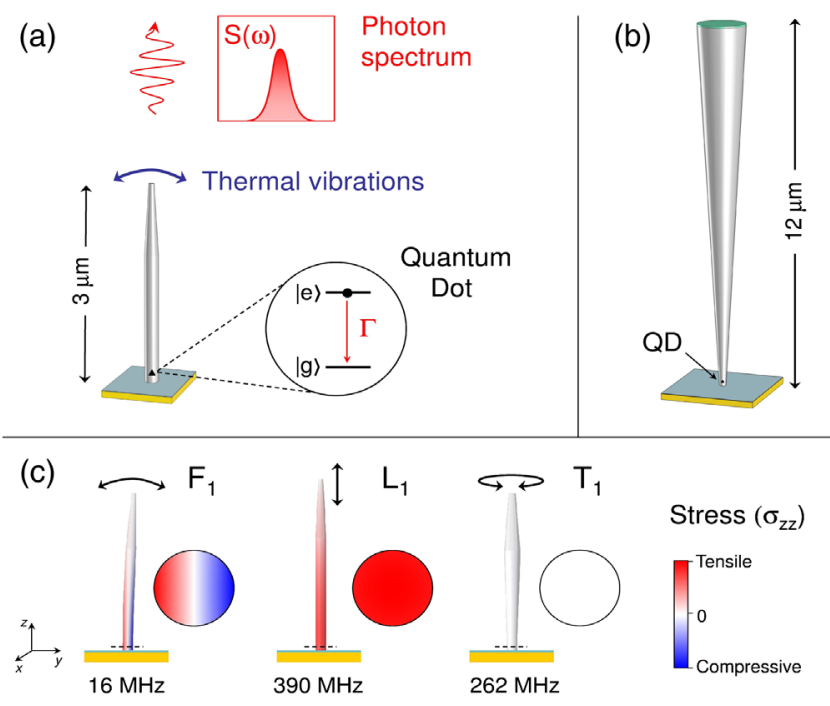

FIG. 1. Nanowire antennas with a "needle" (a) and "trumpet" (b) top taper. The thermal excitation of nanowire vibration modes generates a fluctuating stress which modulates the quantum dot (QD) band gap energy and thus broadens the spectrum $S(\omega)$ of QD photons. (c) Flexural $\left(F_{1}\right)$, longitudinal $\left(L_{1}\right)$ and torsional $\left(T_{1}\right)$ fundamental vibration modes for the needle antenna shown in (a). The color codes the amplitude of the $\sigma_{\mathrm{zz}}$ stress component.

We first evaluate the impact of thermally populated mechanical modes on the QD coherence. The interaction of the QD with electromagnetic modes is treated phenomenologically as a Markovian decay channel. The corresponding spontaneous emission decay rate $\Gamma$ is determined by applying the Fermi golden rule to the photonic environment. This approach holds as long as the density of optical states does not vary significantly over a spectral range which includes relevant phonon modes [see Supplemental Material of Ref. [31]]. While this condition is largely fulfilled for nanowire antennas, narrow band photonic structures (such as resonant microcavities or slowlight waveguides) generally require a more involved treatment $[15,16,32]$.

The QD-phonon coupling arises because mechanical oscillation generates a strain that modulates the QD band gap energy. This interaction is captured by the electronphonon Hamiltonian $\mathcal{H}=\mathcal{H}_{\mathrm{QD}}+\mathcal{H}_{\mathrm{ph}}+\mathcal{C}$. The QD is modeled as a two-level system, with a transition energy $\hbar \omega_{\mathrm{eg}}: \mathcal{H}_{\mathrm{QD}}=\hbar \omega_{\mathrm{eg}}|e\rangle\langle e|$. Compared to the bulk, a nanowire features a set of discrete, low frequency mechanical resonances, typically in the $\mathrm{MHz}-\mathrm{GHz}$ range (the highfrequency cutoff will be discussed later). We focus here on the specific impact of these resonances, keeping in mind that the high-frequency part of the phonon environment also contributes to the spectral broadening of the QD emission (through the formation of acoustic phonon sidebands, and the homogeneous broadening of the zero-phonon line [11]). Neglecting nonlinear intermode couplings [33], the phonon Hamiltonian then reads $\mathcal{H}_{\mathrm{ph}}=$ $\sum_{m} \hbar \omega_{m} b_{m}^{\dagger} b_{m}$, with $\hbar \omega_{m}$ the phonon energy of vibration mode $m$, and $b_{m}^{\dagger}$ the associated phonon creation operator. In the QD-phonon coupling, one retains only the linear term: $\mathcal{C}=\sum_{m} \hbar g_{m}\left(b_{m}+b_{m}^{\dagger}\right)|e\rangle\langle e|$. Since the phonon wavelengths largely exceed the QD size, the latter can be modeled as a pointlike structure, leading to the coupling strength $g_{m}=\left(\partial \omega_{\mathrm{eg}} / \partial u_{m}\right) u_{m}^{\mathrm{zpf}}[34,35]$. For a given mode $m, u_{m}$ is a coordinate describing the nanowire displacement; $u_{m}^{\mathrm{zpf}}$ is the rms amplitude of the associated zero-point fluctuations (details in the Supplemental Material [36]).

Assuming instantaneous excitation in $|e\rangle$, as well as a negligible thermal population of this state, the QD photon emission spectrum $S(\omega)$ can be calculated following Ref. [11]: $S(\omega)=\operatorname{Re} \int_{0}^{\infty} d t e^{-\Gamma t / 2} P(t) e^{-i \omega t}$. In this expression, the dephasing associated with spontaneous emission is introduced phenomenologically. $P(t)=\prod_{m} P_{m}(t)$ describes the time evolution of dipole-dipole correlations driven by the (uncorrelated) contributions of individual vibration modes: $P_{m}(t)=\exp \left[-\theta_{m}^{2} \sin ^{2}\left(\omega_{m} t / 2\right)-\eta_{m}^{2}\left(1-e^{-i \omega_{m} t}\right)\right]$. The argument proportional to $\eta_{m}^{2}=\left(g_{m} / \omega_{m}\right)^{2}$ accounts for phonon emission in the zero-temperature limit. The one proportional to $\theta_{m}^{2}=4 \eta_{m}^{2} N_{m}$, with $N_{m}=\left[\exp \left(\hbar \omega_{m} / k_{B} T\right)-1\right]^{-1}$, accounts for the thermally activated absorption and emission of phonons. For simplicity, we have omitted the so-called polaron shift in $P_{m}(t)$ : it simply induces a rigid spectral shift of the QD emission spectrum. $S(\omega)$ can be recast as the convolution product $S_{\text {rad }}(\omega) * S_{1}(\omega) *$ $S_{2}(\omega) * \cdots ; S_{\text {rad }}(\omega)=(\Gamma / 2) /\left[\omega^{2}+(\Gamma / 2)^{2}\right]$ is the radiatively limited QD emission spectrum, while $S_{m}(\omega)=$ $\int_{-\infty}^{+\infty} d t P_{m}(t) \exp (-i \omega t)$ captures the spectral broadening associated with mode $m$. $S_{m}(\omega)$ consists of spectral lines separated by $\omega_{m}$, whose relative weights are governed by the parameters $\theta_{m}^{2}$ and $\eta_{m}^{2}$.

For pedagogical purposes, we first detail the case of the GaAs needle antenna shown in Fig. 1(a). Its mechanical spectrum is calculated with a finite element software. As schematized in Fig. 1(c), the first vibration modes fall into three families: flexural $(F)$, longitudinal $(L)$, and torsional $(T)$. $T$ modes can be neglected: they essentially generate shear stress, which does not modify the band gap energy of a self-assembled QD, to first order $[19,40]$. In contrast, $F$ and $L$ modes generate a longitudinal stress $\sigma_{\mathrm{zz}}$ which strongly modulates the QD band gap energy [40]. For $L$ modes, $\sigma_{\mathrm{zz}}$ is uniform over horizontal nanowire sections: the coupling strength $g_{m}$ does not depend on the QD lateral position. For $F$ modes, $\sigma_{\mathrm{zz}}$ is null on a central neutral axis, and increases linearly when approaching the sidewall: $g_{m}$ is thus strongly position dependent [41].

Considering a needle antenna cooled down to $T=4 \mathrm{~K}$, $\theta_{m}^{2}$ and $\eta_{m}^{2}$ are plotted in Fig. 2(a) for the first 15 vibration modes. For $F$ modes, we plot the on-sidewall, maximal values. Both $\theta_{m}^{2}$ and $\eta_{m}^{2}$ decrease as the mechanical frequency increases. For all modes $\eta_{m}^{2} \ll 1$, whereas 

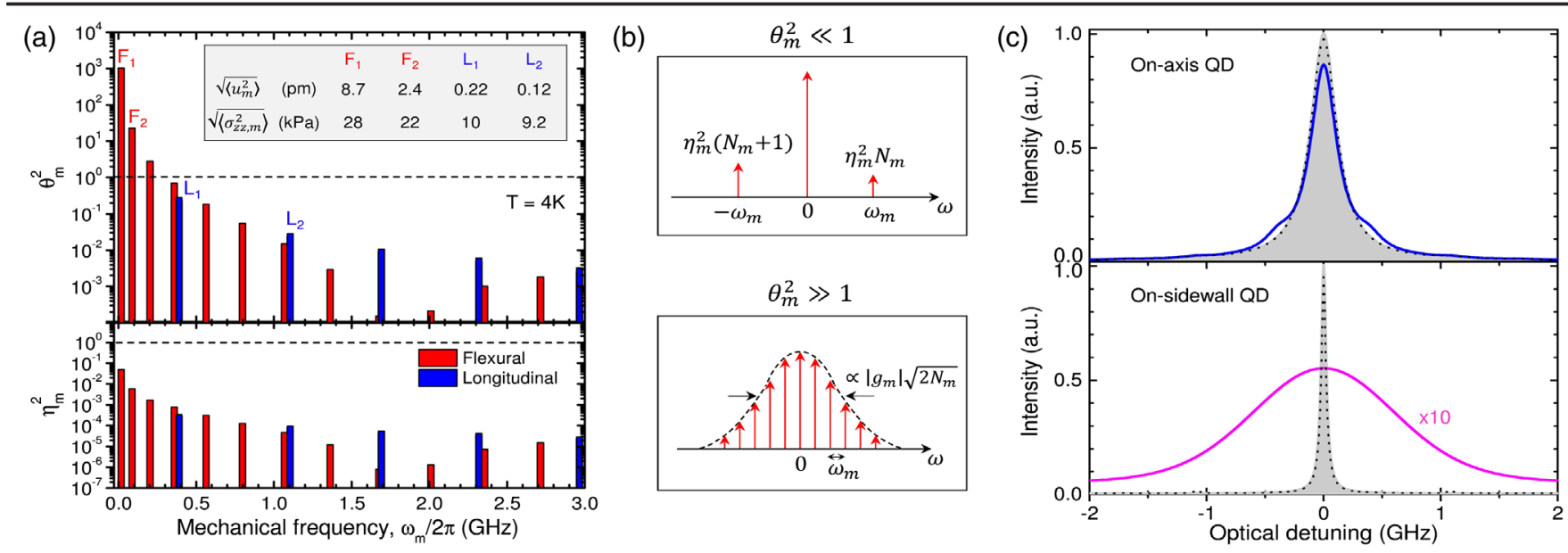

FIG. 2. Impact of the thermal vibrations of a needle antenna at $T=4 \mathrm{~K}$. (a) $\theta_{m}^{2}$ and $\eta_{m}^{2}$ for the first 15 vibration modes. Inset: rms values of the thermal top facet displacement $\left\langle u_{m}^{2}\right\rangle^{1 / 2}$ and of the longitudinal stress $\left\langle\sigma_{\mathrm{zz}, m}^{2}\right\rangle^{1 / 2}$ for a few modes. For $F$ modes, we plot the maximum, on-sidewall values. (b) Line shape $S_{m}(\omega)$ for $\theta_{m}^{2} \gg 1$ and for $\theta_{m}^{2} \ll 1$ (in both cases $\eta_{m}^{2} \ll 1$ ). The arrows represent Dirac $\delta$ peaks. (c) Solid line: Calculated QD emission profile, $S(\omega)$. Dotted line: Calculated QD emission profile without the 7 first mechanical modes ( $6 F$ modes and $1 L$ mode). Filled curve: Lorentzian, radiatively limited emission line, $S_{\text {rad }}(\omega)$. Top panel: on-axis QD; Bottom panel: on-sidewall QD.

$\theta_{m}^{2} \gg 1$ for the first harmonics and $\theta_{m}^{2} \ll 1$ in the high-frequency limit. For the first vibration modes $\left(\theta_{m}^{2} \gg 1 \gg \eta_{m}^{2}\right), S_{m}(\omega)$ is a comb of spectral lines with a Gaussian envelope having a standard deviation $\left|g_{m}\right| \sqrt{2 N_{m}}$ [see Fig. 2(b)]. In this case, the QD experiences a spectral broadening which directly reflects the thermally driven stress fluctuations whose rms values are given for a few modes as an inset in Fig. 2(a). Since $\left|g_{m}\right| \sqrt{2 N_{m}}$ features a rather smooth dependence with respect to $\omega_{m}$, all modes found in this limit significantly contribute to the spectral broadening. The high-frequency limit corresponds to a perturbative regime $\left(\eta_{m}^{2}, \theta_{m}^{2} \ll 1\right)$. As shown in Fig. 2(b), $S_{m}(\omega)$ is then composed of a central spectral line, flanked by a red and a blue sideband, whose weights are $\eta_{m}^{2}\left(N_{m}+1\right)$ and $\eta_{m}^{2} N_{m}$, respectively. For the considered needle antenna, we compute the mechanical spectrum up to $8 \mathrm{GHz}$, and the sideband weight for the higher frequency modes is below $10^{-4}$.

Figure 2(c) shows the calculated QD emission spectrum for on-axis and on-sidewall locations, at $T=4 \mathrm{~K}$. For reference, we plot as a shaded curve the radiatively limited spectra. These spectra differ significantly, because the local density of optical states varies in the nanowire section (Ref. [29] and Supplemental Material [36]). We take here $\Gamma=1.74 \times \Gamma_{\text {bulk }}$ on the axis, and $\Gamma=0.33 \times \Gamma_{\text {bulk }}$ on the sidewall [42], with $\Gamma_{\text {bulk }}=(1 \mathrm{~ns})^{-1}=2 \pi \times 160 \mathrm{MHz}$ the reference decay rate of a typical QD embedded in bulk GaAs. Because of nanowire vibrations, both on-axis and on-sidewall emitters feature a spectrum that differs from the radiatively limited line shape. An on-axis emitter is only coupled to $L$ modes; the sidebands generated by $L_{1}$ (390 MHz) are clearly resolved in the spectrum. On the sidewall, the emitter experiences in addition a strong contribution of low-frequency $F$ modes. The QD emission is then well approximated by a Voigt profile, which is much broader than the radiatively limited line shape. Similar results are obtained in photonic trumpets, for which QD linewidths have been measured with resonant spectroscopy. At low driving power, the QD investigated in Ref. [19] features a full width at half maximum (FWHM) of $5.1 \mu \mathrm{eV}$ and a radiatively limited FWHM of $0.7 \mu \mathrm{eV}$. The QD position was determined by strain-gradient mapping [41]: the emitter is off axis by $35 \mathrm{~nm}$ (the mean wire radius is $145 \mathrm{~nm}$ ). Our theory then predicts a FWHM of $2.9 \mu \mathrm{eV}$, showing that even for QDs that are relatively close to the nanowire axis, thermal vibrations constitute a major source of decoherence at $T=4 \mathrm{~K}$.

Figure 2(c) also demonstrates that the spectral broadening is dominated by the contribution of the first mechanical modes. By suppressing the first 7 modes in a needle antenna, one restores an emission profile which is very close to the radiatively limited one. This can be directly traced back to the rapid decrease of $\theta_{m}^{2}$ and $\eta_{m}^{2}$ at large mechanical frequency.

For quantitative analysis, we now consider the photon indistinguishability $I$, a figure of merit which corresponds to the outcome of a two-photon coalescence experiment, a key phenomenon for many quantum information protocols. Assuming a perfectly antibunched photon emission $\left[g^{2}(0)=0\right]$, it can be calculated as in Refs. [28,43]: $I=\Gamma \int_{0}^{\infty} d t e^{-\Gamma t}|P(t)|^{2}$. The numbers given in the following include only the effect of nanowire low-frequency vibrations, and exclude all other decoherence channels. Figure 3 confirms that at $T=4 \mathrm{~K}$, thermal vibrations of needle and trumpet antennas lead to indistinguishability values that are not compatible with the requirements of quantum optics experiments. 


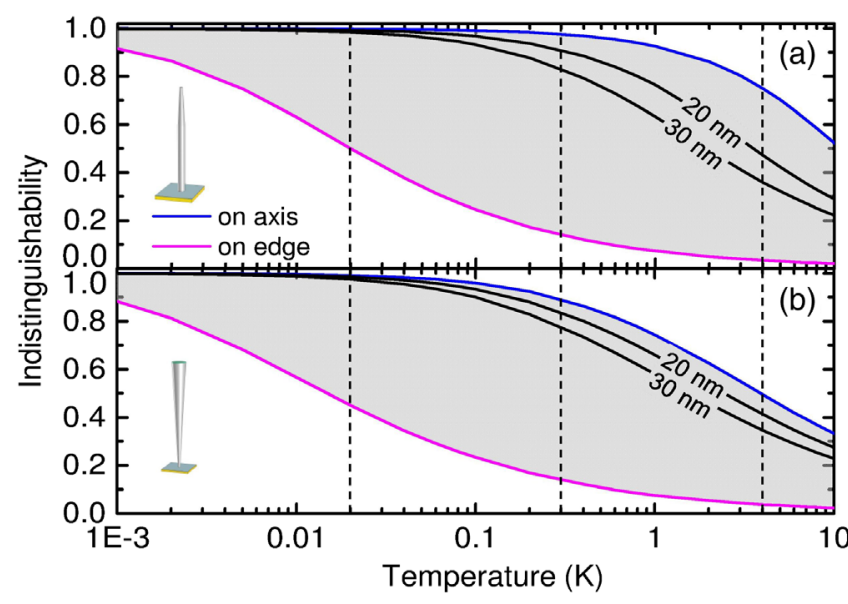

FIG. 3. Photon indistinguishability $I$ as a function of the operation temperature $T$ for a typical needle (a) and trumpet (b) nanowire antenna. We consider four transverse QD positions: on axis, $20 \mathrm{~nm}$ of axis, $30 \mathrm{~nm}$ of axis, and on sidewall. The vertical dashed lines indicate the typical base temperature in a dilution fridge $(20 \mathrm{mK})$, a pumped ${ }^{3} \mathrm{He}$ cryostat $(300 \mathrm{mK})$ and a liquid ${ }^{4} \mathrm{He}$ cryostat $(4 \mathrm{~K})$.

To improve $I$, a first obvious strategy is to reduce the temperature. In a pumped ${ }^{3} \mathrm{He}$ cryostat $(T=300 \mathrm{mK})$, a QD located on the axis of a needle antenna features $I=0.98$ and emitters with a distance to the axis below $20 \mathrm{~nm}$ exhibit $I>0.92$ [Fig. 3(a)]. However, on-sidewall emitters are still plagued with a poor $I$. In this situation, accurate emitter positioning in the nanowire section is critical to minimize vibration decoherence. For top-down devices, this could be realized with deterministic lithography techniques [44-46]. Interestingly, the bottom-up fabrication route defines an on-axis QD with an even better accuracy [21]. Operating the source in a dilution fridge $(T=20 \mathrm{mK}$ ) further improves $I$ and the tolerance on the emitter lateral position.

Below we explore a different (and less expensive) path: we propose to engineer the decoherence channels, bringing all vibration modes deep into the perturbative regime $\left(\theta_{m}^{2}\right.$, $\eta_{m}^{2} \ll 1$ ), for which mechanical sidebands feature a vanishingly small weight. This approach largely suppresses the impact of low-frequency vibrations, restoring photon indistinguishability when the antenna is operated in a standard ${ }^{4} \mathrm{He}$ cryostat. Generally speaking, it is interesting to shift mechanical resonances towards the high frequency domain, because both $N_{m}$ and $\eta_{m}$ decrease. A second important improvement direction is to locate the emitter as far as possible from high-stress zones. In the following, we propose three designs which exploit separately or combine these ideas. The values of $\theta_{m}$ and $\eta_{m}$ which quantitatively support the associated discussions are given in the Supplemental Material [36]. The Supplemental Material also details the photonic rationale of the designs, which preserve large light extraction efficiency $(>80 \%)$. Changes in the geometry also imply (small) changes in the QD

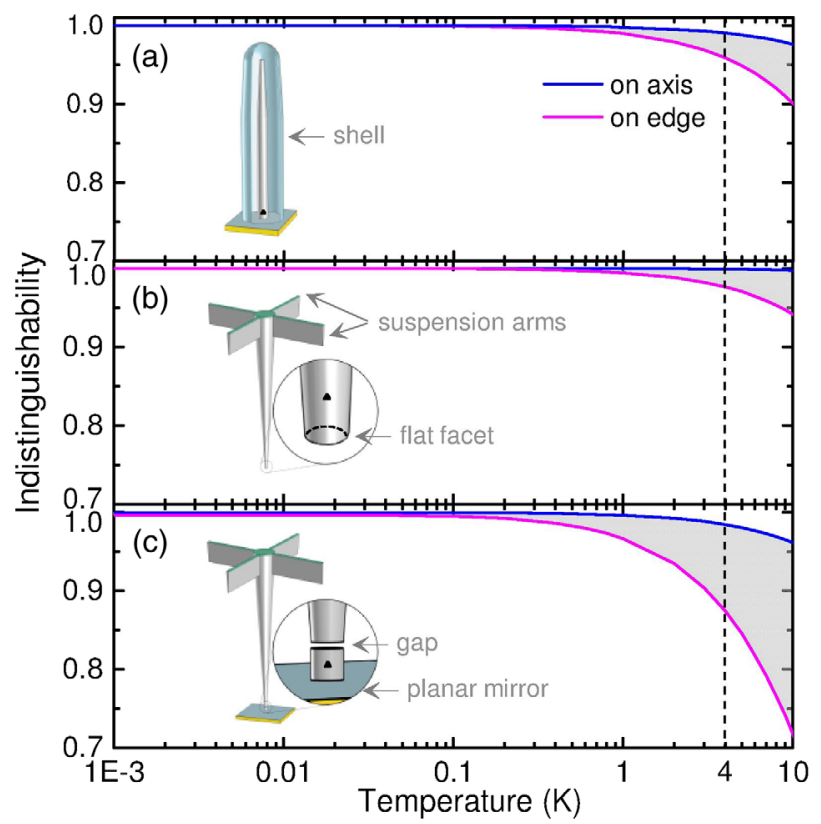

FIG. 4. Photon indistinguishability $I$ as a function of the operation temperature $T$ for three mechanically engineered antennas. (a) Needlelike antenna covered with a conformal, low-index dielectric shell (thickness of $500 \mathrm{~nm}$ ). (b) Suspended antenna. (c) Mechanically decoupled top taper. In (b) and (c) the suspension arms are connected to large pillars (not shown).

emission rate $\Gamma$, which are taken into account in the calculation of $I$.

As illustrated in Fig. 4(a), covering the antenna with a conformal dielectric shell made of a low-index material represents a simple yet effective solution to suppress vibration decoherence. We consider here $\mathrm{SiO}_{2}$, but other materials could be employed as well. A shell with a thickness of just $s=500 \mathrm{~nm}$ leads to excellent performance at $T=4 \mathrm{~K}$ : An on-axis emitter features $I=0.99$, and $I$ exceeds 0.96 at any QD lateral positions. The shell largely suppresses the impact of $F$ modes, because an emitter embedded in the core remains far from the lateral stress maximum, which is located on the shell sidewall. In addition, the frequency of $F$ modes increases with the structure diameter. The impact of $L$ modes also decreases, essentially because the shell increases the effective mass of the resonator, henceforth reducing $g_{m}$. As $s$ further increases, performance continues to improve to match the one of bulk material. From a technological point of view, the limit $s \rightarrow \infty$ can be conveniently achieved through a planarization of the device. This approach also proves to be very efficient when applied to trumpetlike antennas.

In standard nanowire antennas, QDs are located close to a longitudinal maximum of the vibration-induced strain. The suspended photonic trumpet shown in Fig. 4(b) completely reverses this situation. In the proposed device, the top part of the taper is anchored to distant pillars and the QD is located close to the bottom part of the nanowire, which is terminated by a flat facet, used as a mirror. The emitter is thus located 
close to a free end of the nanowire, which dramatically decreases the coupling strengths and restores excellent spectral properties $(I>0.98$ for all QD positions). Suspended trumpets have been recently fabricated [47], and could be alternatively realized by gluing the top facet of the trumpet on the cleaved end of a single-mode fiber [48]. In both cases the flat bottom facet could be conveniently realized with a sacrificial layer.

A last possible strategy is to shift all mechanical resonances to very large $(\mathrm{GHz})$ values. In the device schematized in Fig. 4(c), a $10 \mathrm{~nm}$ thin gap is introduced between a $200 \mathrm{~nm}$-high bottom nanowire section and the top taper. The gap is sufficiently thin to minimize the optical discontinuity, while enabling perfect mechanical decoupling of the bottom part from the taper. Owing to its nanoscale volume, the bottom section exhibits a fundamental resonance frequency as high as $2 \mathrm{GHz}$, which represents an increase of more than 3 orders of magnitude compared to a standard trumpet. This strategy also performs very well: $I$ reaches 0.98 on the wire axis and exceeds 0.87 for all emitter positions. As discussed in the Supplemental Material [36], such a gap can be defined by etching a sacrificial layer.

To conclude, we have demonstrated that nanowire thermal vibrations constitute a major decoherence channel for an embedded QD. This limitation can be overcome through specific engineering of the mechanical properties of the nanowire antenna. Our work establishes such nanomechanical engineering as a crucial part of the design of highperformance light-matter interfaces based on photonic nanostructures. In particular, we anticipate that mechanical vibrations also play an important role in suspended on-chip waveguides $[8,49]$, a potential building block of integrated quantum photonic circuits.

The authors gratefully acknowledge fruitful discussions with A. Auffèves, L. Besombes, E. Denning, J. Mørk, and J.P. Poizat, and the support of Y. Curé for finite element simulations. This work was supported by ANR Project QDOT (ANR-16-CE09-0010-01) and by the CEA DRFImpulsion project SOUPAPE. S. K. acknowledges funding from the European Union's Horizon 2020 research and innovation programme under the Marie Sklodowska-Curie Grant Agreement No. 754303, and the Agence Nationale de la Recherche under the programme "Investissements d'avenir" (ANR-15-IDEX-02). P. V. acknowledges support from the ERC Starting Grant No. 758794 Q-ROOT.

*julien.claudon@cea.fr

${ }^{\dagger}$ These authors contributed equally to this work.

[1] J. L. O'Brien, A. Furusawa, and J. Vučković, Nat. Photonics 3, 687 (2009).

[2] A. V. Kuhlmann, J. Houel, A. Ludwig, L. Greuter, D. Reuter, A. D. Wieck, M. Poggio, and R. J. Warburton, Nat. Phys. 9, 570 (2013).
[3] Y.-M. He, Y. He, Y.-J. Wei, D. Wu, M. Atatüre, C. Schneider, S. Höfling, M. Kamp, C.-Y. Lu, and J.-W. Pan, Nat. Nanotechnol. 8, 213 (2013).

[4] A. V. Kuhlmann, J. H. Prechtel, J. Houel, A. Ludwig, D. Reuter, A. D. Wieck, and R. J. Warburton, Nat. Commun. 6, 8204 (2015).

[5] X. Ding, Y. He, Z.-C. Duan, N. Gregersen, M.-C. Chen, S. Unsleber, S. Maier, C. Schneider, M. Kamp, S. Höfling, C.-Y. Lu, and J.-W. Pan, Phys. Rev. Lett. 116, 020401 (2016).

[6] N. Somaschi, V. Giesz, L. De Santis, J. C. Loredo, M. P. Almeida, G. Hornecker, S. L. Portalupi, T. Grange, C. Antón, J. Demory, C. Gómez, I. Sagnes, N. D. LanzillottiKimura, A. Lemaître, A. Auffèves, A. G. White, L. Lanco, and P. Senellart, Nat. Photonics 10, 340 (2016).

[7] F. Liu, A. J. Brash, J. O'Hara, L. M. P. P. Martins, C. L. Phillips, R. J. Coles, B. Royall, C. Clarke, E. Bentham, N. Prtljaga, I. E. Itskevich, L. R. Wilson, M. S. Skolnick, and A. M. Fox, Nat. Nanotechnol. 13, 835 (2018).

[8] H. Thyrrestrup, G. Kiršanskè, H. Le Jeannic, T. Pregnolato, L. Zhai, L. Raahauge, L. Midolo, N. Rotenberg, A. Javadi, R. Schott, A. D. Wieck, A. Ludwig, M. C. Löbl, I. Söllner, R. J. Warburton, and P. Lodahl, Nano Lett. 18, 1801 (2018).

[9] L. Besombes, K. Kheng, L. Marsal, and H. Mariette, Phys. Rev. B 63, 155307 (2001).

[10] I. Favero, G. Cassabois, R. Ferreira, D. Darson, C. Voisin, J. Tignon, C. Delalande, G. Bastard, P. Roussignol, and J.-M. Gérard, Phys. Rev. B 68, 233301 (2003).

[11] E. A. Muljarov and R. Zimmermann, Phys. Rev. Lett. 93, 237401 (2004).

[12] P. Borri, W. Langbein, U. Woggon, V. Stavarache, D. Reuter, and A. D. Wieck, Phys. Rev. B 71, 115328 (2005).

[13] A. J. Ramsay, A. V. Gopal, E. M. Gauger, A. Nazir, B. W. Lovett, A. M. Fox, and M. S. Skolnick, Phys. Rev. Lett. 104, 017402 (2010).

[14] T. Jakubczyk, V. Delmonte, S. Fischbach, D. Wigger, D. E. Reiter, Q. Mermillod, P. Schnauber, A. Kaganskiy, J.-H. Schulze, A. Strittmatter, S. Rodt, W. Langbein, T. Kuhn, S. Reitzenstein, and J. Kasprzak, ACS Photonics 3, 2461 (2016).

[15] T. Grange, N. Somaschi, C. Antón, L. De Santis, G. Coppola, V. Giesz, A. Lemaître, I. Sagnes, A. Auffèves, and P. Senellart, Phys. Rev. Lett. 118, 253602 (2017).

[16] J. Iles-Smith, D. P. S. McCutcheon, A. Nazir, and J. Mørk, Nat. Photonics 11, 521 (2017).

[17] A. Reigue, J. Iles-Smith, F. Lux, L. Monniello, M. Bernard, F. Margaillan, A. Lemaitre, A. Martinez, D. P. S. McCutcheon, J. Mørk, R. Hostein, and V. Voliotis, Phys. Rev. Lett. 118, 233602 (2017).

[18] P. Lodahl, S. Mahmoodian, and S. Stobbe, Rev. Mod. Phys. 87, 347 (2015).

[19] M. Munsch, A. V. Kuhlmann, D. Cadeddu, J.-M. Gérard, J. Claudon, M. Poggio, and R. J. Warburton, Nat. Commun. 8, 76 (2017).

[20] J. Claudon, J. Bleuse, N. S. Malik, M. Bazin, P. Jaffrennou, N. Gregersen, C. Sauvan, P. Lalanne, and J.-M. Gérard, Nat. Photonics 4, 174 (2010).

[21] M.E. Reimer, G. Bulgarini, N. Akopian, M. Hocevar, M. B. Bavinck, M. A. Verheijen, E. P. A. M. Bakkers, L. P. Kouwenhoven, and V. Zwiller, Nat. Commun. 3, 737 (2012). 
[22] M. Munsch, N. S. Malik, E. Dupuy, A. Delga, J. Bleuse, J.-M. Gérard, J. Claudon, N. Gregersen, and J. Mørk, Phys. Rev. Lett. 110, 177402 (2013).

[23] P. E. Kremer, A. C. Dada, P. Kumar, Y. Ma, S. Kumar, E. Clarke, and B. D. Gerardot, Phys. Rev. B 90, 201408(R) (2014).

[24] M. A. M. Versteegh, M. E. Reimer, K. D. Jöns, D. Dalacu, P. J. Poole, A. Gulinatti, A. Giudice, and V. Zwiller, Nat. Commun. 5, 5298 (2014).

[25] T. Huber, A. Predojević, M. Khoshnegar, D. Dalacu, P. J. Poole, H. Majedi, and G. Weihs, Nano Lett. 14, 7107 (2014).

[26] M. Khoshnegar, T. Huber, A. Predojević, D. Dalacu, M. Prilmüller, J. Lapointe, X. Wu, P. Tamarat, B. Lounis, P. Poole, G. Weihs, and H. Majedi, Nat. Commun. 8, 15716 (2017).

[27] G. Lindwall, A. Wacker, C. Weber, and A. Knorr, Phys. Rev. Lett. 99, 087401 (2007).

[28] P. Tighineanu, C. L. Dreessen, C. Flindt, P. Lodahl, and A. S. Sørensen, Phys. Rev. Lett. 120, 257401 (2018).

[29] J. Claudon, N. Gregersen, P. Lalanne, and J.-M. Gérard, ChemPhysChem 14, 2393 (2013).

[30] I. Friedler, C. Sauvan, J. P. Hugonin, P. Lalanne, J. Claudon, and J.-M. Gérard, Opt. Express 17, 2095 (2009).

[31] D. P. S. McCutcheon and A. Nazir, Phys. Rev. Lett. 110, 217401 (2013).

[32] K. Roy-Choudhury and S. Hughes, Optica 2, 434 (2015).

[33] D. Cadeddu, F. R. Braakman, G. Tütüncüoglu, F. Matteini, D. Rüffer, A. Fontcuberta i Morral, and M. Poggio, Nano Lett. 16, 926 (2016).

[34] I. Wilson-Rae, P. Zoller, and A. Imamoğlu, Phys. Rev. Lett. 92, 075507 (2004).

[35] I. Yeo, P.-L. de Assis, A. Gloppe, E. Dupont-Ferrier, P. Verlot, N. S. Malik, E. Dupuy, J. Claudon, J.-M. Gérard, A. Auffèves, G. Nogues, S. Seidelin, J.-P. Poizat, O. Arcizet, and M. Richard, Nat. Nanotechnol. 9, 106 (2014).

[36] See Supplemental Material at http://link.aps.org/ supplemental/10.1103/PhysRevLett.123.247403 for the calculation of $g_{m}$, the photonic properties of the devices, and details on mechanical simulations. The Supplemental Material includes Refs. [37-39].
[37] I. Vurgaftman, J. R. Meyer, and L. R. Ram-Mohan, J. Appl. Phys. 89, 5815 (2001).

[38] T. Häyrynen, J. R. de Lasson, and N. Gregersen, J. Opt. Soc. Am. A 33, 1298 (2016).

[39] T. Heindel, C. Schneider, M. Lermer, S. H. Kwon, T. Braun, S. Reitzenstein, S. Höfling, M. Kamp, and A. Forchel, Appl. Phys. Lett. 96, 011107 (2010).

[40] P. Stepanov, M. Elzo-Aizarna, J. Bleuse, N. S. Malik, Y. Curé, E. Gautier, V. Favre-Nicolin, J.-M. Gérard, and J. Claudon, Nano Lett. 16, 3215 (2016).

[41] P.-L. de Assis, I. Yeo, A. Gloppe, H. A. Nguyen, D. Tumanov, E. Dupont-Ferrier, N. S. Malik, E. Dupuy, J. Claudon, J.-M. Gérard, A. Auffèves, O. Arcizet, M. Richard, and J.-P. Poizat, Phys. Rev. Lett. 118, 117401 (2017).

[42] J. Bleuse, J. Claudon, M. Creasey, N. S. Malik, J.-M. Gérard, I. Maksymov, J.-P. Hugonin, and P. Lalanne, Phys. Rev. Lett. 106, 103601 (2011).

[43] A. Kiraz, M. Atatüre, and A. Imamoğlu, Phys. Rev. A 69, 032305 (2004).

[44] A. K. Nowak, S. L. Portalupi, V. Giesz, O. Gazzano, C. Dal Savio, P.-F. Braun, K. Karrai, C. Arnold, L. Lanco, I. Sagnes, A. Lemaître, and P. Senellart, Nat. Commun. 5, 3240 (2014).

[45] M. Gschrey, A. Thoma, P. Schnauber, M. Seifried, R. Schmidt, B. Wohlfeil, L. Krüger, J. H. Schulze, T. Heindel, S. Burger, F. Schmidt, A. Strittmatter, S. Rodt, and S. Reitzenstein, Nat. Commun. 6, 7662 (2015).

[46] L. Sapienza, M. Davanco, A. Badolato, and K. Srinivasan, Nat. Commun. 6, 7833 (2015).

[47] Q. Mermillod, T. Jakubczyk, V. Delmonte, A. Delga, E. Peinke, J.-M. Gérard, J. Claudon, and J. Kasprzak, Phys. Rev. Lett. 116, 163903 (2016).

[48] D. Cadeddu, J. Teissier, F. Braakman, N. Gregersen, P. Stepanov, J.-M. Gérard, J. Claudon, R. J. Warburton, M. Poggio, and M. Munsch, Appl. Phys. Lett. 108, 011112 (2016).

[49] M. N. Makhonin, J. E. Dixon, R. J. Coles, B. Royall, E. Clarke, M. S. Skolnick, and A. M. Fox, Nano Lett. 14, 6997 (2014). 